\title{
A Computer-Aided System for Malignancy Risk Assessment of Nodules in Thyroid US Images based on Boundary Features
}

\author{
Michalis Savelonas, Dimitris Maroulis and Manolis Sangriotis \\ Department of Informatics and Telecommunications, University of Athens, \\ Athens, Greece \\ \{msavel, dmaroulis, sagri\}@di.uoa.gr
}

\begin{abstract}
In this paper, a novel computer-based approach is proposed for malignancy risk assessment of thyroid nodules in ultrasound images. The proposed approach is based on boundary features and is motivated by the correlation which has been addressed in medical literature between nodule boundary irregularity and malignancy risk. In addition, local echogenicity variance is utilized so as to incorporate information associated with local echogenicity distribution within nodule boundary neighborhood. Such information is valuable for the discrimination of high-risk nodules with blurred boundaries from medium risk nodules with regular boundaries. Analysis of variance is performed, indicating that each boundary feature under study provides statistically significant information for the discrimination of thyroid nodules in ultrasound images, in terms of malignancy risk. $k$-nearest neighbor and support vector machine classifiers are employed for the classification tasks, utilizing feature vectors derived from all combinations of features under study. The classification results are evaluated with the use of the receiver operating characteristic. It is derived that the proposed approach is capable of discriminating between medium-risk and high-risk nodules, obtaining an area under curve, which reaches 0.95 .
\end{abstract}

Keywords: Computer-Aided Diagnosis, Ultrasound, Thyroid Nodules, Boundary Features.

\section{Introduction}

The results of clinical research demonstrate that the presence of blurred or irregular thyroid nodule boundaries on ultrasound (US) images correlate with malignancy risk [1],[2]. In this light, the quantification of nodule boundary irregularity by boundary-based features could be valuable for malignancy risk assessment, contributing to the objectification of medical decisions. Such boundary-based features could be combined with intensity and textural information within an integrated computer-aided-diagnosis (CAD) tool.

Previous attempts on CAD categorization of thyroid nodules on US images include evaluation of parameters from the gray level histogram of thyroid US images [3],[4], intensity features extracted by the utilization of Radon transform [5], textural features extracted from gray level spatial-dependence matrices [6],[7], and the application of discriminant 
analysis [3],[6].

\subsection{Medical background}

Thyroid nodules are lumps within the thyroid gland, with a considerable clinical importance because of the risk of malignancy, involving occurrences of papillary, follicular, medullary and anaplastic carcinomas. The prevalence of thyroid nodules increases with age, extending to more than $50 \%$ of the world's population, whereas $50 \%$ of people with solitary nodules detected by experienced physicians have additional nodules detected when examined further by ultrasonography [8]-[11]. The US grading of nodules proposed by Tomimori et al [1], classifies nodules in four grades associated with an increasing risk for malignancy:

1) Grade I: small round and anechoic area, suggestive of a thyroid cyst.

2) Grade II: isoechoic or hyperechoic solid nodules with or without cystic change and coarse calcification, suggestive of adenomatous goiter. Isoechoic solid nodule or complex nodule, are suggestive of follicular adenoma.

3) Grade III: i) hypoechoic solid nodule with regular boundary may represent a follicular neoplasm, ii) cystic nodule with solid component may represent a papillary carcinoma. These nodule types shall be referred as medium-risk nodules within the text.

4) Grade IV: hypoechoic solid nodule with an irregular boundary and with the presence of calcifications is considered suspicious for malignancy and highly suggestive of thyroid carcinoma. These nodule types shall be referred as highrisk nodules within the text.

Figure 1 illustrates four thyroid US images containing nodules associated with Grades I-IV.

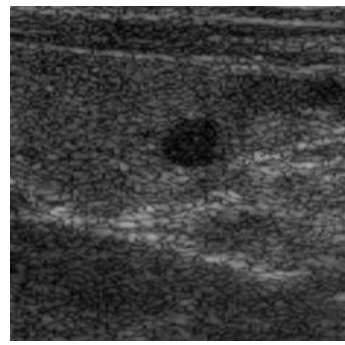

(a)

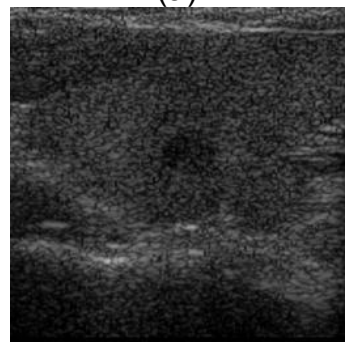

(c)

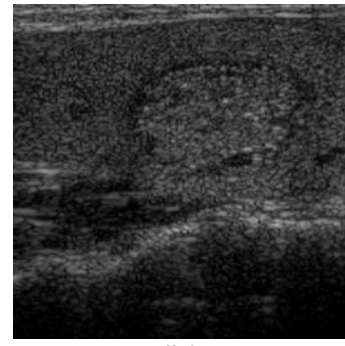

(b)

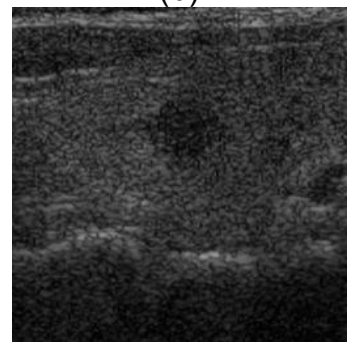

(d)

Fig. 1. Thyroid US images containing nodules associated with: (a) grade I, (b) grade II, (c) grade III, and (d) grade IV, according to the US grading proposed by Tomimori et al [1]. 


\subsection{Boundary features}

Chain Coding (CC), introduced by Freeman [12], is a common boundary representation approach. Various applications using CC representation have been reported up to date [13]-[15], however, to the best of our knowledge, $\mathrm{CC}$ or CC-derived boundary features have not been considered for the definition of boundary features of US findings. This could be attributed to the consideration that, since every small deviation along the boundary is encoded, CC is expected to be extremely sensitive to US speckle noise.

The recent development of noise-robust methods for the delineation of nodules in thyroid US images encourages the application of CC-derived boundary features, such as CC histogram itself [16] and compactness (CMP), on US findings. Compactness (CMP) is a typical measure of the space-filling nature of a boundary. A high CMP value is associated with an irregular boundary, since it indicates a large perimeter enclosing a small area. Typically, benign masses are expected to have lower values of CMP as compared to malignant tumors [17]. Kallergi [18] suggests CMP as a discriminative boundary feature for assessing malignancy risk of breast nodules.

Other boundary features applied in medical image analysis include the normalized radial length (NRG) [19], the area ratio [20], the fractal dimension (FD) [21],[31], the roughness index, the spiculation index, and the fractional concavity [23]. In addition, the extraction of boundary features by analyzing spectral domain, as it is the case with Fourier descriptor or wavelet descriptor, has been proved to provide noise-robust boundary representation in various applications [31]. An extensive review on boundary features can be found in [25].

The main limitation of the aforementioned features, with the exception of NRG, is that they are exclusively derived from the boundary of a finding, as produced by segmentation or manual delineation. Therefore, they do not encode local echogenicity information within the boundary neighborhood. Intuitively, such information could be connected to blurred nodule boundaries, reportedly associated with thyroid malignancy risk. The NRG, which is a measure of the average orientation of the gray level gradients along the boundary, provides such local intensity information; however, the calculations of image gradients involved are susceptive to US speckle noise. A noise-robust locally calculated image feature defined so as to encode this local intensity information would effectively indicate the existence of highrisk nodules, covering a "middle ground" between region-based and pure boundary features.

The focus of this paper is to investigate CAD approaches for the discrimination between medium-risk and high-risk nodules in US images. Local echogenicity variance (LEV) is defined and utilized, aiming to the enhancement of the classification performance in cases of US image datasets, which include high-risk nodules characterized by blurred boundaries. Moreover, according to the US grading of Tomimori et al [1], some high-risk nodules can be discriminated from medium-risk nodules by the irregularity of their boundaries. This indicates that boundary features, although not sufficient, could be useful within the context of a nodule classification scheme. Based on the results of 
previous research which support the utilization of CMP and FD as boundary features [18],[21],[22] this paper investigates their discriminative capability. The utilization of CMP aims to encode large-scale irregularity information as it is the existence of lobes, whereas FD is utilized so as to quantify small-scale irregularity. The discriminative capability of each boundary feature is statistically analyzed by means of analysis of variance (ANOVA). The $k$-nearest neighbor classifier ( $k$-NN) is used so as to facilitate direct comparisons between various boundary feature combinations by means of receiver operating characteristic (ROC) curves. The classification performance is further optimized by the use of support vector machine (SVM) classifiers. It should be noted that the proposed computerbased approach is the first utilizing nodule boundary features for thyroid malignancy risk assessment.

The rest of this paper is organized in four sections. Section 2 presents the boundary features utilized by the proposed approach, followed by LEV. The results of the application of the proposed approach for malignancy risk estimation of thyroid nodules in US images are apposed in Section 3, and the conclusions of this study are summarized in Section 4.

\section{Materials and Methods}

The proposed computer-based approach utilizes CMP and FD which are pure boundary features, involving calculations based on the shape of the nodule boundary. CMP captures large-scale irregularity information as it is the existence of lobes, whereas FD captures small-scale irregularity information. In addition, LEV is introduced and utilized by the proposed approach so as to enhance the capability of discriminating nodules characterized by blurred boundaries.

\subsection{Feature extraction}

The core idea of CC involves the movement along a digital curve or along a sequence of border pixels based on $n$ connectivities. The direction of each movement is encoded by means of a numbering scheme $\{i \mid i=0,1, \ldots n\}$, which denotes an angle of 360xiln, clockwise or counter-clockwise, from the positive $x$-axis, as shown in Fig.2. Thus, CC can be viewed as a connected sequence of straight-line segments with specified lengths and directions. 

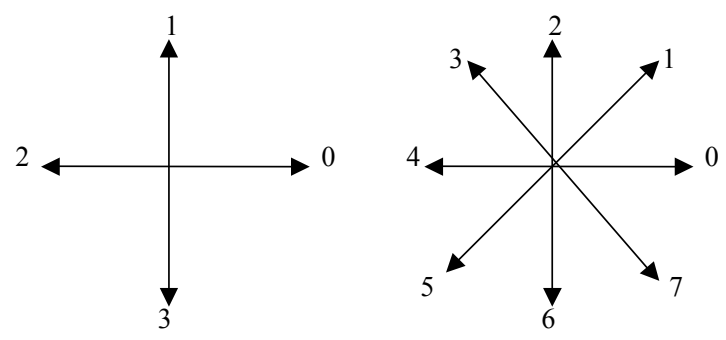

Fig. 2. Directions of 4-directional and 8-directional CC.

The CC can be normalized with respect to the starting point by a straightforward procedure: it is treated as a circular sequence of direction numbers and the starting point is redefined so that the resulting sequence of numbers forms an integer of minimum magnitude. Normalization with respect to rotation can be obtained by using the first difference of the CC instead of the code itself. This difference is obtained by counting the number of direction changes that separate two adjacent elements of the code. For instance, the first difference of the 4-direction chain code 10103322 is 3133030 . The code can be treated as a circular sequence by computing the first element of the difference using the transition between the last and first components of the chain. Accordingly, the result in this example is 33133030 [26]. The histogram describing the frequency of occurrence of each CC element could be used as a boundary feature, as proposed by livarinen and Visa [16]. The chain code histogram $(\mathrm{CCH})$ is computed as:

$$
p(k)=n_{k} / n
$$

where $n_{k}$ is the number of chain code values $k$ in a CC and $n$ is the number of links in a CC. The CCH reflects the probabilities of different directions present in a boundary. Moreover, since a CC is a complete representation of a curve, it can be used to calculate boundary features of an object, such as perimeter, area and CMP.

CMP is a dimensionless quantity providing a measure of contour complexity, whereas it is independent of translation, rotation, and scale. For this study, the following definition of CMP is adopted:

$$
C M P=\frac{P^{2}}{A}
$$

where $P$ is the perimeter and $A$ is the area of the object, calculated with the use of CC. According to eq. (2), CMP has a minimum value of $4 \pi$ and increases for elongated shapes or shapes with rough contours. As CMP is derived from $P$ and $A$, which are global boundary features, it encodes large-scale irregularity information, as the existence of lobes or protrusions. 


\subsection{Fractal dimension}

Fractals are self-similar structures characterized by a non-integer dimension. Their geometrical complexity can be quantified by means of the fractal dimension (FD). FD is an elegant well-defined multi-scale measure that has been shown to possess a strong correlation with human's intuitive notion of roughness and has been widely applied to different problems in image processing and pattern recognition [27]. It can be computed utilizing limiting processes, such as the box counting algorithm, which is based on partitioning the shape image into square boxes of size $L \times L$ and counting the number of boxes $(n(L))$ containing a portion of the shape. The FD is calculated as the absolute value of the slope of the line obtained from the linear regression of the $(\log (L), \log (n(L)))$ curve, by varying the box size $L$ (Fig. 3). However, FD computation using real, discrete data, such as images, is not obvious. Much of the literature regarding fractal analysis has been concerned with the estimation of FD given a discrete data set [22].

FD is capable of encoding small-scale irregularity information associated with nodule boundaries. Microlobulated or highly spiculated contours of malignant tumors are expected to demonstrate fractal behavior, which should be absent in the case of benign masses [21],[22]. Rangayyan and Nguyen [21] computed FD from the one-dimensional signature of a given mass, defined as the Euclidean distance of each contour point from the centroid of the contour.

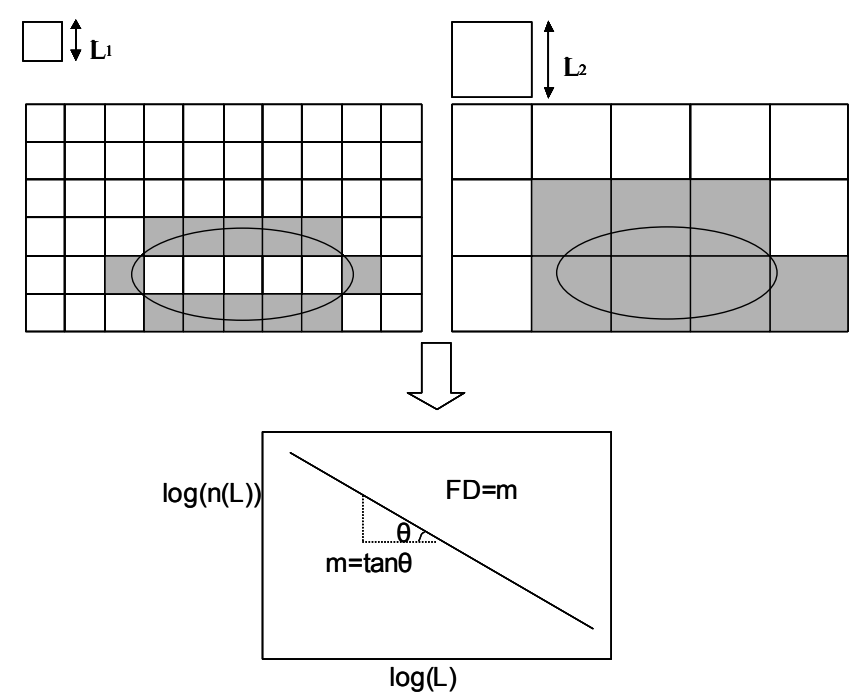

Fig. 3. Illustration of the box counting method.

\subsection{Local echogenicity variance}

The US grading of thyroid nodules introduced by Tomimori et al [1] suggests that both medium-risk and high-risk nodules are associated with hypoechogenicity. However, high-risk nodules are also associated with blurred, i.e. poorly-defined, or irregular boundaries. Figure 4 illustrates examples of medium-risk nodules (Fig. 4a), high-risk 
nodules (Fig. 4b) with blurred boundaries and high-risk nodules with irregular boundaries (Fig. 4c). Figure 5 illustrates the respective delineations obtained by the application of the variable background active contour (VBAC) model [28]. It can be observed that in cases of high-risk nodules characterized by irregular boundaries, the derived delineations are actually irregular (Fig. 5c). Such nodule cases are expected to be differentiable from medium-risk nodules by the utilization of pure boundary features. However, in the cases of high-risk nodules characterized by blurred boundaries, the derived delineations are as smooth (Fig. 5b) as the delineations of medium-risk nodules (Fig. 5a). Accordingly, their discrimination cannot be solely based on boundary features quantifying boundary irregularity.

Aiming to incorporate information associated with local echogenicity distribution within nodule boundary neighborhood and enhance the accuracy of the proposed nodule classification approach, we employ local echogenicity variance (LEV) calculated within a stripe centered on the available delineation of each nodule. This stripe contains all pixels within a small distance from the delineation, so as to capture local intensity variations and cover a "middle ground" between region-based and pure boundary features. LEV values are lower within neighborhoods of blurred nodule boundaries, as opposed to the high LEV values obtained in cases of boundaries well defined by abrupt echogenicity changes. The utilization of LEV facilitates the discrimination of high-risk nodules characterized by blurred boundaries from medium-risk nodules.

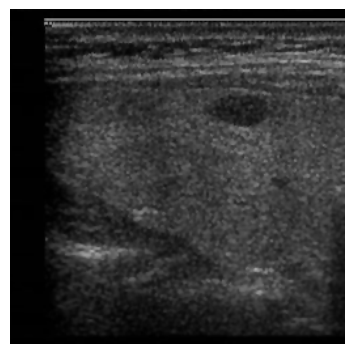

(a)

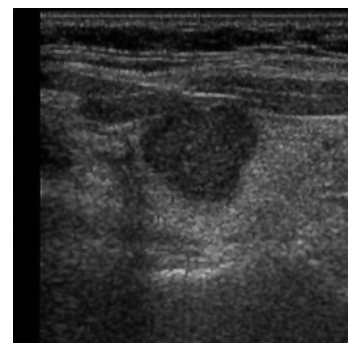

(b)

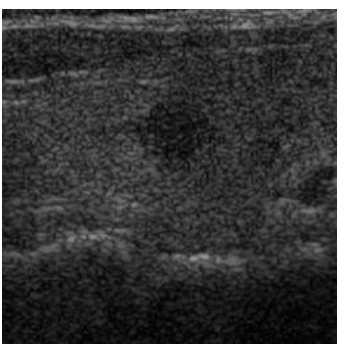

(c)

Fig. 4. Nodule examples associated with: (a) medium risk, (b) high risk characterized by blurred boundaries, and (c) high risk characterized by irregular boundaries.

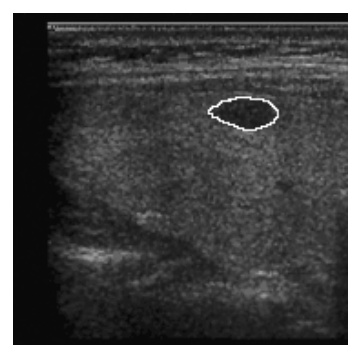

(a)

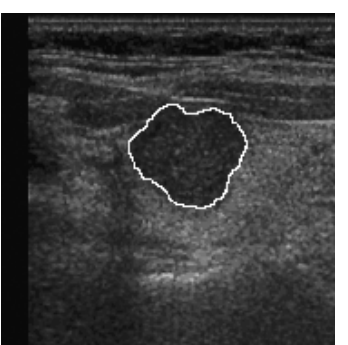

(b)

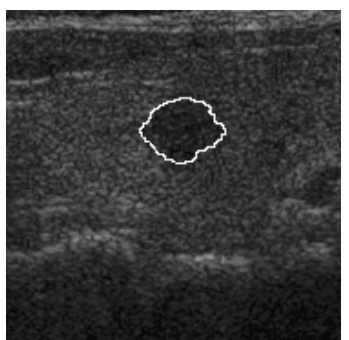

(c)

Fig. 5. Delineations of nodules illustrated in Fig. 4, obtained by the application of the VBAC model.

Thyroid US examinations were performed on 387 patients between October 2003 and September 2006 for evaluation of suspected thyroid nodular disease, using a digital US imaging system HDI 3000 ATL with a 5-12 MHz 256 grey-level depth, linear transducer in the radiology department of Euromedica diagnostic center in Greece. Time 
gain compensation setting had a linear increasing gain compared to the depth. Instrument settings were set accordingly to the built-in 'SmallPartTest' Philips protocol, magnification was set to 1:1, and dynamic range was set to $150 \mathrm{~dB} / \mathrm{C} 4$. The criteria for referral were suspicion of the presence of one or more thyroid nodules by physical exam or the presence of an "incidental" nodule discovered by an imaging technique. A total of 173 longitudinal digital images of hypoechoic thyroid nodule cases were acquired by retrospectively reviewing the records of patients. The study is limited to cases of hypoechoic nodules, since hypoechogenicity is associated with medium-risk and high-risk thyroid nodules [1]. The VBAC model [28] is applied for the delineation of the thyroid nodules. The VBAC model constants used in the experiments were chosen as: $\lambda^{+}=5, \lambda^{-}=5, \mu=650$ and $a=10^{-13}$, as suggested by Maroulis et al [28]. The width of the stripe associated with the calculation of LEV is set to 7 pixels.

\section{Results}

Three expert physicians characterized the boundary of each hypoechoic nodule used in the experiments as regular (grade III), blurred or irregular (grade IV), following the classification of Tomimori et al. "Ground truth" characterizations are produced according to a majority rule so as to ameliorate the subjectivity induced by each expert. Malignancy risk estimation based on nodules' appearance in US is acceptable in medical practice [1],[9]. However, the results of this work cannot directly be compared to works such as [6], where the "ground truth" is derived by histological findings. The derived US image dataset is comprised of 96 cases of medium-risk nodules, 41 cases of high-risk nodules with blurred boundaries and 34 cases of high-risk nodules with irregular boundaries. A special purpose software suite in Microsoft Visual C++ was developed and executed on a $3.2 \mathrm{GHz}$ Intel Pentium IV workstation for feature extraction and $k$-NN classification. The implementation of the SVMs was based on the publicly available libSVM library [29].

The experimental results are organized in two main parts: the first part is investigating the statistical significance of the boundary features under study for the discrimination of medium risk nodules from high-risk nodules with irregular or blurred boundaries. The second part is dedicated to the evaluation of the classification performance of all combinations of the boundary features under study.

\subsection{Statistical analysis}

Analysis of variance (ANOVA) is a general technique for evaluating the statistical significance of the difference of independent data groups with respect to a measured characteristic. ANOVA involves the so-called null hypothesis testing, where the null hypothesis is that the means among the groups are equal, under the assumption that the sampled populations are normally distributed [30]. 
Table 1 illustrates the results of analysis of variance for CMP, FD and LEV for the discrimination of medium-risk nodules from high-risk nodules with blurred boundaries (experiment 1), as well as from high-risk nodules with irregular boundaries (experiment 2).

A $p$-value of less than 0.05 was yielded for LEV in experiment 1 , indicating a statistically significant difference of this feature when it comes to nodule cases of different boundary smoothness. This is in agreement with the definition of LEV as a boundary smoothness index. On the contrary, the results obtained for CMP and FD demonstrated that varying boundary smoothness does not significantly differentiate ( $p$-value $>>0.05)$ the value of these features. This can be attributed to the fact that both CMP and FD are defined as boundary irregularity indexes, whereas both nodule types of experiment 1 are characterized by relatively regular boundaries.

The results of experiment 2 were predictably opposite, demonstrating statistically significant differences for CMP and FD ( $p$-value<0.05), and not for LEV ( $p$-value $>>0.05)$. These results can be justified by similar arguments with experiment 1 , considering that in the case of experiment 2 nodule classes are characterized by different boundary irregularity.

The results of both experiments indicate that all features under study encode boundary information which can be valuable for the characterization of thyroid nodules in terms of malignancy risk. In addition, it can be observed that CMP is more discriminative than FD, obtaining a lower $p$-value in all experiments. This indicates that CMP-encoded large-scale nodule boundary irregularity, which is associated with lobes or protrusions, is more indicative of malignancy risk than small-scale boundary irregularity, which is more sensitive to US speckle and image resolution.

Table 1

p-values obtained for LEV, CMP and FD for experiments 1 and 2.

\begin{tabular}{ccc}
\hline Feature & $p$-value (experiment 1) & $p$-value (experiment 2) \\
\hline LEV & 0.02 & 0.24 \\
CMP & 0.29 & 0.01 \\
FD & 0.34 & 0.03 \\
\hline
\end{tabular}

\subsection{Classification}

The $k$-nearest neighbor $(k-N N)$ is a well-known nonparametric classifier with essentially one free parameter, the number of neighbors $k$, which can be optimized by a leave-one-out estimate of the error rate $k$ [31]. $k$-NN classification involves the computation of the distances between a test pattern and all patterns in the training set, whereas the associated decision rule is based on the minimum distance.

The $k$-NN classifier, with $k=5$, is utilized for the comparison of the classification performance obtained by various combinations of CMP, FD and LEV. This classifier facilitates direct comparisons, as it does not depend on multiple 
parameters. The value of $k$ was selected considering the classification results obtained in preliminary experiments. It should be noted that high-risk nodules, either with blurred or irregular boundaries are classified as positive, whereas medium-risk nodules are classified as negative. In accordance with the recommendation of Brandley [32], 10-fold cross validation was performed for the generation of ROC curves. Table 2 illustrates the 3 highest values of area under curve (AUC) derived from combinations of CMP, FD and LEV. The highest AUC is obtained by the combined use of CMP, FD and LEV, indicating that each of these features provides complementary boundary information.

Table 2

The 3 highest values of AUC derived from combinations of CMP, FD and LEV.

\begin{tabular}{lc}
\hline Feature & AUC \\
\hline CMP, FD, LEV & 0.93 \\
CMP, LEV & 0.91 \\
FD, LEV & 0.89 \\
\hline
\end{tabular}

Figure 6 illustrates the ROC curve obtained by the $k$-NN classifier utilizing the combination of all boundary features. The 95\% confidence interval for AUC, calculated following the approach described in [33], is [0.88, 0.96]. It can be derived that for specificity equal to 0.80 , the obtained sensitivity is 0.94 . The associated confusion matrix is illustrated in Table 3.

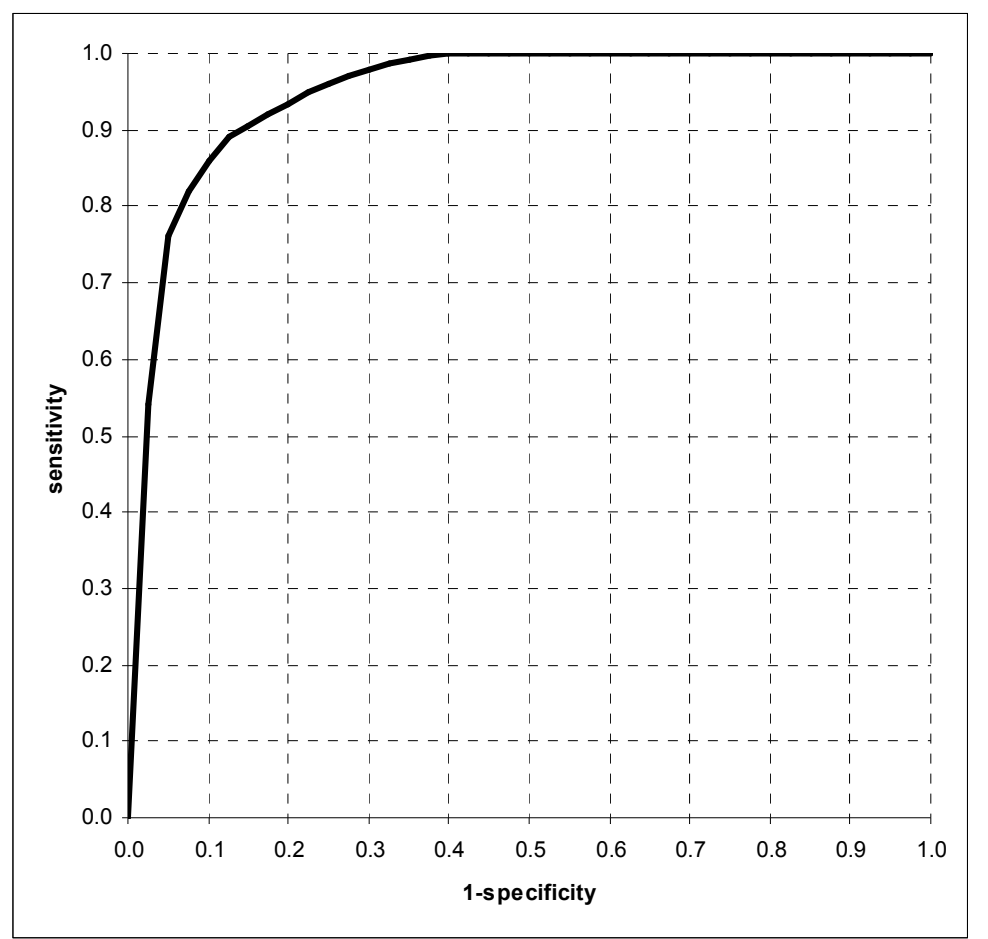

Figure 6. ROC curve obtained by $k$-NN utilizing the combination of all boundary features. 
Table 3

Confusion matrix obtained by $k-\mathrm{NN}$ utilizing the combination of all boundary features.

\begin{tabular}{llccc}
\hline & & \multicolumn{3}{c}{$k-N N$} \\
\cline { 2 - 4 } & & medium-risk & high-risk (blurred) & high-risk (irregular) \\
\hline \multirow{3}{*}{ expert } & medium-risk & 75 & 18 & 3 \\
& high-risk (blurred) & 4 & 37 & 0 \\
& high-risk (irregular) & 0 & 0 & 34 \\
\hline
\end{tabular}

The classification performance of the proposed approach was further optimized by the use of the SVM classifier [34].

SVM is formulated so as to satisfy an optimization criterion associated with the width of the margin between the classes, i.e. the empty area around the decision boundary defined by the distance to the nearest training patterns. These patterns, called support vectors, define the classification function, whereas their number is minimized by maximizing the margin [31].

The value of the tolerance parameter $C$ and the kernel type [34] providing the maximum classification accuracy, were experimentally determined as $C=40$ and the $3^{\text {rd }}$ order polynomial kernel respectively. 10 -fold cross validation was also used in this case. Figure 7 illustrates the ROC curve obtained by the SVM classifier utilizing the combination of all boundary features. The AUC obtained is 0.95 , which is $2 \%$ higher when compared to the AUC obtained by using $k$-NN, whereas the associated 95\% confidence interval calculated following the approach described in [33], is [0.91, 0.97]. In addition, it is derived that for specificity equal to 0.80 , the obtained sensitivity is 0.98 . The associated confusion matrix is illustrated in Table 4. It can be observed that the utilization of the SVM classifier reduced the number of high-risk nodules with blurred boundaries which were classified as medium-risk, when compared to the results obtained with the use of the $k$-NN classifier. 


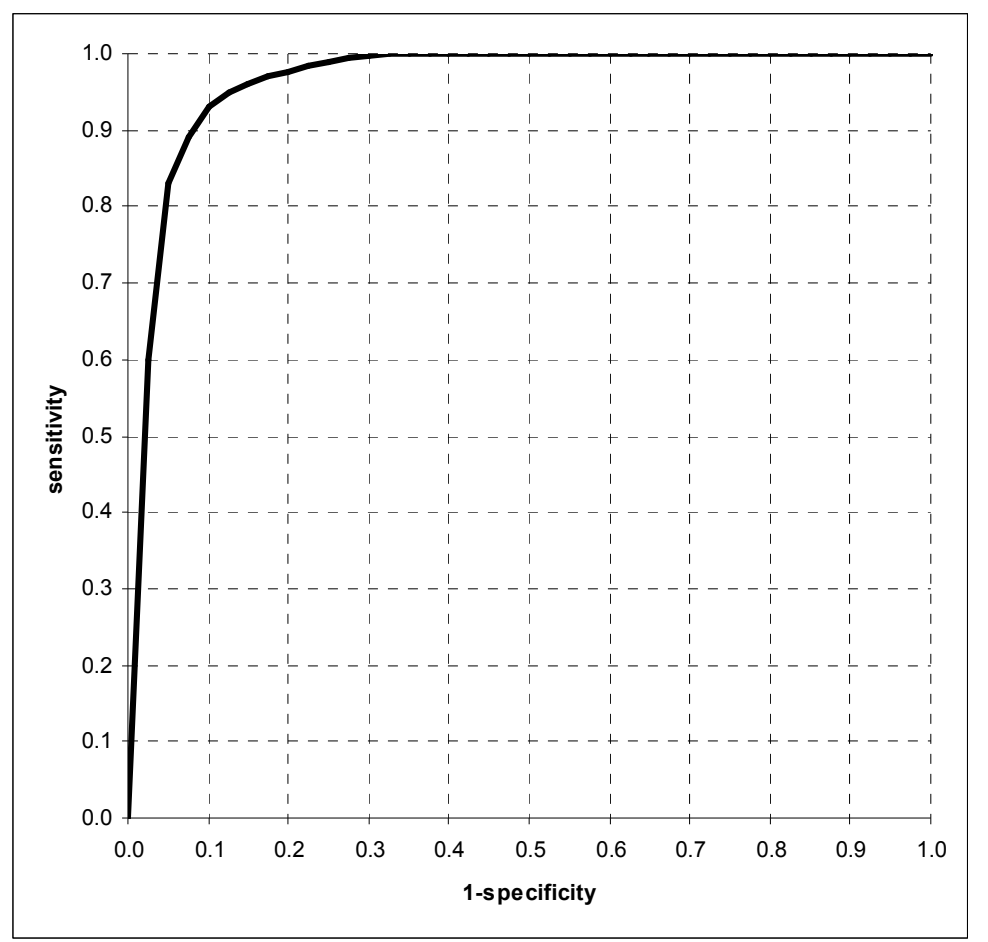

Figure 7. ROC curve obtained by SVM utilizing the combination of all boundary features.

Table 4

Confusion matrix obtained by SVM utilizing the combination of all boundary features.

\begin{tabular}{clccc}
\hline & & \multicolumn{3}{c}{ SVM } \\
\cline { 2 - 5 } & & medium-risk & high-risk (blurred) & high-risk (irregular) \\
\hline \multirow{3}{*}{ expert } & medium-risk & 75 & 18 & 3 \\
& high-risk (blurred) & 1 & 40 & 0 \\
& high-risk (irregular) & 0 & 0 & 34 \\
\cline { 2 - 5 } & & &
\end{tabular}

It should be noted that the utilized features do not describe shape variability in general, but the aspects of shape variability which correlate with nodule malignancy risk according to medical literature. This is in agreement with the results of additional experiments, which do not demonstrate a measurable improvement by embedding features such as concavity or axial symmetry [35] within the utilized feature set. This could be attributed to the fact that these features are correlated with compactness.

\section{Conclusions}

In this paper, we introduced a novel computer-based approach for malignancy risk assessment of thyroid nodules in US images. The proposed approach is based on compactness (CMP) and fractal dimension (FD), as well as on local echogenicity variance (LEV), which is defined so as to incorporate information associated with local echogenicity distribution within nodule boundary neighborhood. Such information allows the discrimination of high-risk nodules with blurred boundaries from medium risk nodules with regular boundaries. ANOVA was performed to evaluate the statistical significance of the utilized features. In addition, $k$-NN and SVM classifiers where employed for the 
classification tasks, utilizing feature vectors derived from all combinations of boundary features under study.

The experimental evaluation on real thyroid US images lead to the following conclusions:

1. CMP, FD, and LEV, all provide statistically significant information for the discrimination of thyroid nodules in US images in terms of malignancy risk.

2. The optimal classification performance is obtained by the combined use of all boundary features under study, indicating that each of these features provides complementary boundary information.

3. The utilization of the SVM classifier leads to higher classification performance, when compared to the one obtained with the use of $k-\mathrm{NN}$.

4. The proposed approach is capable of discriminating between medium-risk and high-risk nodules, contributing to the objectification of the diagnostic process.

Future perspectives of this work include the derivation of textural information, as well as information extracted from video frame sequences of thyroid US, aiming at an integrated medical decision support system for the identification of thyroid nodules.

\section{Acknowledgments}

This work was partially funded by National and Kapodestrian University of Athens, Special Account of Research Grants. We would like to thank the Departments of Radiology of EUROMEDICA S.A. and Henry Dunant Hospital for the provision of high quality anonymized data as well as for their assistance in the assessment of the thyroid nodules.

\section{Conflict of Interest Statement}

None declared.

\section{References}

[1] Tomimori, E.K., Camargo, R.Y., Bisi, H., Medeiros-Neto, G., 1999. Combined ultrasonographic and cytological studies in the diagnosis of thyroid nodules, Biochimie $81(5), 447-452$.

[2] Koike, E., Noguchi, S., Yamashita, H., Murakami, T., Ohshima, A., Kawamato, H., Yamashita, H., 2001. Ultrasonographic characteristics of thyroid nodules: prediction of malignancy, Arch.Surg.136, $334-337$.

[3] Hirning, T., Zuna I., Schlaps D., et al., 1989. Quantification and classification of echographics findings in the thyroid gland by computerized Bmode texture analysis. Eur. J. Radiol., 9, $244-247$. 
[4] Mailloux, G., Bertrand, M., Stampfler, R., Ethier, S., 1986. Computer analysis of echographic textures in Hashimoto disease of the thyroid. J. Clin. Ultr., 14, 521-527.

[5] Savelonas, M.A., lakovidis, D.K., Dimitropoulos, N., Maroulis, D., 2007. Computational characterization of thyroid tissue in the Radon domain, Proc. IEEE Int. Symp. Comp.-Bas. Med. Syst. (CBMS), 189-192.

[6] Müller, M.J., Lorenz, D., Zuna, I., Lorenz, W.J., van Kaick, G.,1989. The value of computer-assisted sonographic tissue characterization in focal lesions of the thyroid, Der Radiologe, 29, 132-136.

[7] Tsantis, S., Cavouras, D., Kalatzis, I., Piliouras, N., Dimitropoulos, N., Nikiforidis, G., 2005. Development of a support vector machine-based image analysis system for assessing the thyroid nodule malignancy risk on ultrasound, Ultr. Med. Biol., 31 (11), 1451-1459.

[8] Feld, S. et al, 1996. AACE clinical practice guidelines for the diagnosis and management of thyroid nodules, End. Pract., 78-84.

[9] Frates, M.C., Benson, C.B., Charboneau, J.W. et al., 2005. Management of thyroid nodules detected at US: society of radiologists in ultrasound consensus conference statement. Radiology, 237 (3), 794 - 800.

[10] Mihmanli, I., Kantarci, F., 2006. Concurrent routine breast and thyroid sonography for detection of thyroid tumors, Am. J. Roentgenol., 187 (4), W448 - W448.

[11] Frates, M.C., Benson, C.B., Doubilet, P.M. et al., 2006. Prevalence and distribution of carcinoma in patients with solitary and multiple thyroid nodules on sonography, J. Clin. End. Metabol., 91 (9), 3411 - 3417.

[12] Freeman, H.,1961. On the encoding of arbitrary geometric configurations, IRE Trans. Electron. Comput. EC-10, 260-268.

[13] Liu, Y.K., Zalik, B., 2005. An efficient chain code with Huffman coding, Patt. Rec. 38, 553-557.

[14] Zingaretti, P., Gasparroni, M., Vecci, L., 1998. Fast chain coding of region boundaries, IEEE Trans. Patt. Anal.Mach.Intell.20 (4), 407 -414.

[15] Shih, F.Y., Wong, W.T., 1999. A one-pass algorithm for local symmetry of contours from chain code, Patt. Rec., 32 (7), 1203 -1210.

[16] livarinen, J., Visa, A., 1996. Shape recognition of irregular objects, Proc. SPIE 2904, D.P. Casasent (Ed.), Intelligent Robots and Computer Vision XV: Algorithms, Techniques, Active Vision, and Materials Handling, 25-32.

[17] Nakamura, K. et al, 2000. Computerized analysis of the likelihood of malignancy in solitary pulmonary nodules with use of artificial neural networks, Radiol. 14 (3), 823-830.

[18] Kallergi, M., 2004. Computer-aided diagnosis of mammographic microcalcification clusters, Med. Phys. 31 (2), 314-326.

[19] Chou, Y.H. et al, 2001. Stepwise logistic regression analysis of tumor contour features for breast ultrasound diagnosis, Ultr. Med. Biol. 27 (11), 1493-1498.

[20] Loncaric, S., 1998. A survey of shape analysis techniques, Patt. Rec. 31 (8) (1998) 983-1001.

[21] Rangayyan, R.M., Nguyen, T.M., 2007. Fractal analysis of contours of breast masses in mammograms, J. Dig. Im. 20 (3), $223-237$.

[22] Tao, Y., Lam, E.C.M., Tang, Y., 2001. Feature extraction using wavelet and fractal, Patt. Rec. Lett. 22, 271-287.

[23] Rangayyan, R.M., El-Faramawy, N.M., Desautels, J.E.L., Alim, O.A., 1997. Measures of acutance and shape for classification of breast tumors. IEEE Trans. Med. Imag. 16 (6), 799-810.

[24] Georgiou, H., Mavroforakis, M., Dimitropoulos, N., Cavouras, D., Theodoridis, S., 2007. Multi-scaled morphological features for the characterization of mammographic masses using statistical classification schemes, Art. Intell. Med. 41, 39-55.

[25] Zhang, D., Lu, G., 2004. Review of shape representation and description techniques, Patt. Rec. 37, 1-19.

[26] Gonzales, R.C., Woods, R.E., 2008. Digital image processing, 3rd edition, Prentice Hall.

[27] Peitgen, H.O., Saupe, D., 1998. The science of fractal images, Springer-Verlag.

[28] Maroulis, D.E., Savelonas, M.A., lakovidis, D.K., Karkanis, S.A., Dimitropoulos, N., 2007. Variable background active contour model for computer-aided delineation of nodules in thyroid ultrasound images, IEEE Trans. Inf. Tech. Biom. 11 (5), 537-543.

[29] Chang, C.C., Lin, C.J., 2001. LIBSVM : a library for support vector machines. Software available at http://www.csie.ntu.edu.tw/ cjlin/libsvm. 
[30] Theodoridis, S., Koutroumbas, K., 2006. Pattern Recognition, 3rd edition, Academic Press.

[31] Jain, A.K., Duin, R.P.W., Mao, J., 2000. Statistical pattern recognition: a review, IEEE Trans. Patt. Anal. Mach. Intell., 22, (1), 4-37.

[32] Brandley, A.P., 1997. The use of the area under the ROC curve in the evaluation of machine learning algorithms. Patt. Rec., 30 (7), 11451159.

[33] Stalbovskaya, V., Hamadicharef, B., Ifeachor, E., 2007. Sample size determination using ROC analysis, Proc. Int. Conf. Comp. Intell. Med. Health. (CIMED).

[34] Scholkopf, T.F.B., 1997. Support vector learning. Springer-Verlag.

[35] Atallah, J.R., 1985. On symmetry detection, IEEE Trans. Comp., 663-666. 